\title{
Common neural patterns for serial order coding in working memory, number and letter domains: A multi-voxel pattern analysis approach
}

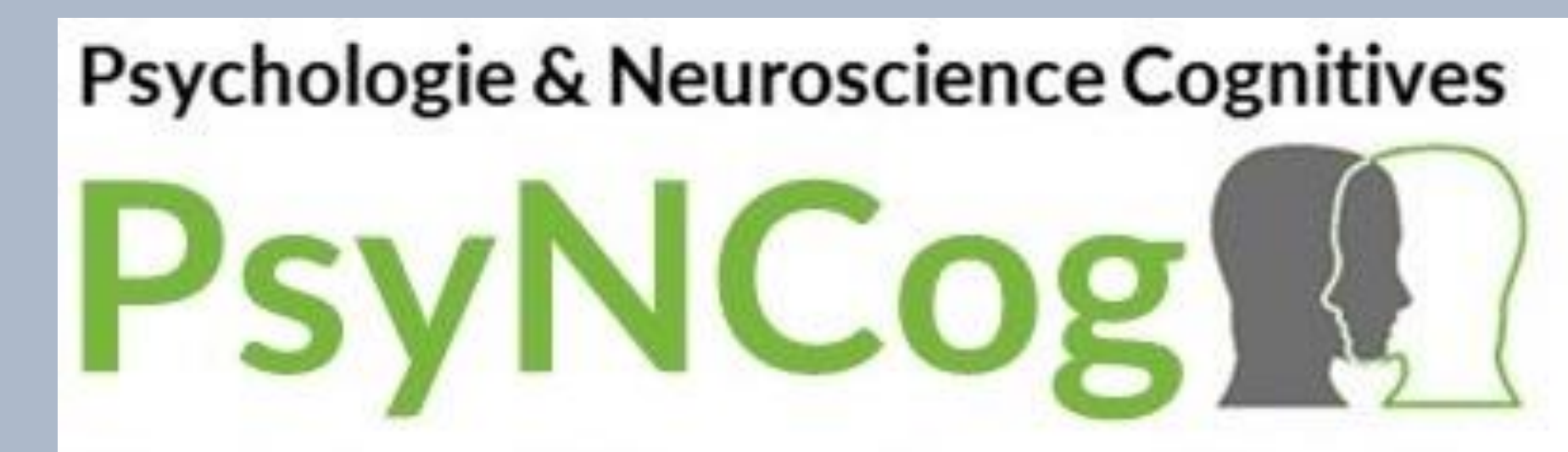

Psychology \& Neuroscience of Cognition

LEROY Nathan a , ATTOUT Lucie ${ }^{a, b, c}$, MAJERUS Steve a,b,c

a University of Liège, Belgium

b Psychology and Neuroscience of Cognition Research Unit (PsyNCog), Belgium

c Fund for Scientific Research (FNRS), Belgium

\section{Introduction}

The retention of serial order information allows for the structured encoding and maintenance of the sequential order of events and stimuli (Majerus \& Attout, 2018). It's a fundamental component of working memory potentially associated with numerical and alphabetical abilities and critical for success in many different cognitive tasks. The codes used to represent serial order remain however poorly understood.

\section{Aim}

We assessed the hypothesis that serial order information is coded using domain general ordinal representations that support serial order coding also in other domains characterized by ordinal distance effects such as numbers and letters.

\section{Method}

Participants: 25 healthy young adults (15 women) (mean : 23.48 years \pm 2.9 )

Tasks: Three ordinal judgement tasks with further manipulation of ordinal distance effects, and a luminance comparison control task.

\section{Alphabetical task}

"Alphabetical order?»

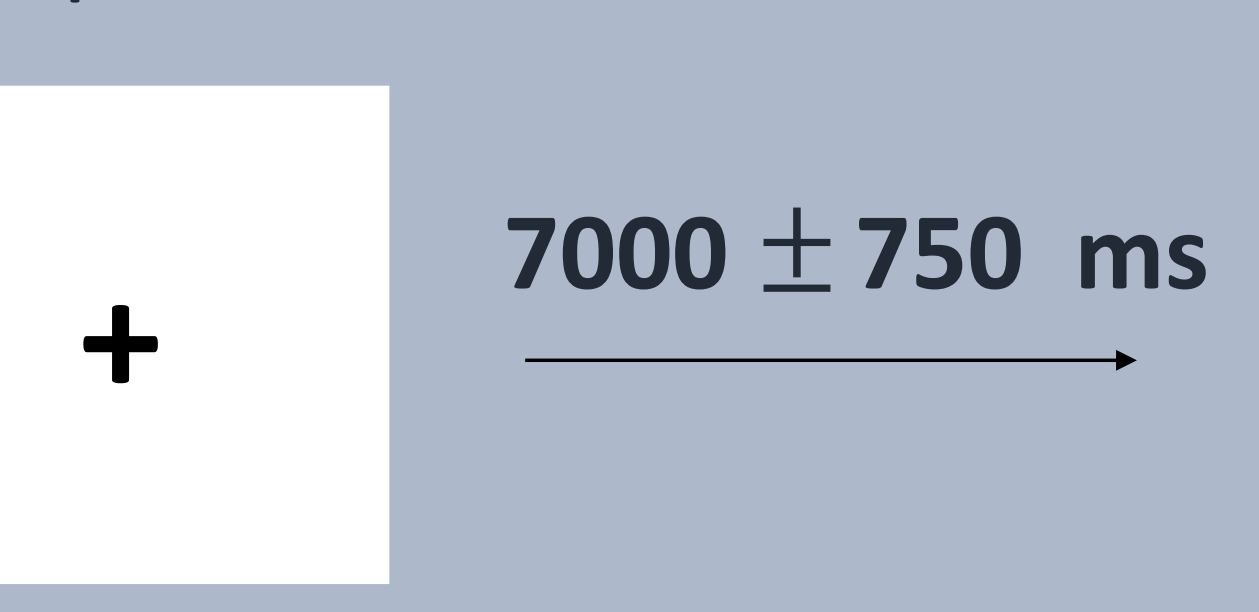

\begin{tabular}{cc} 
& $\begin{array}{l}\text { Distance : } 1 \text { or } \mathbf{2} \\
\text { Order : ascending or descending }\end{array}$ \\
Probe $6 \mathrm{~s}$ & \\
\hline $\mathrm{F}$ & Exemple of : \\
$\mathrm{G}$ & Distance 1 \\
$\mathrm{H}$ & Ascending \\
\hline
\end{tabular}

\section{Numerical task}

« Numerical order? »$$
\text { (1) }
$$

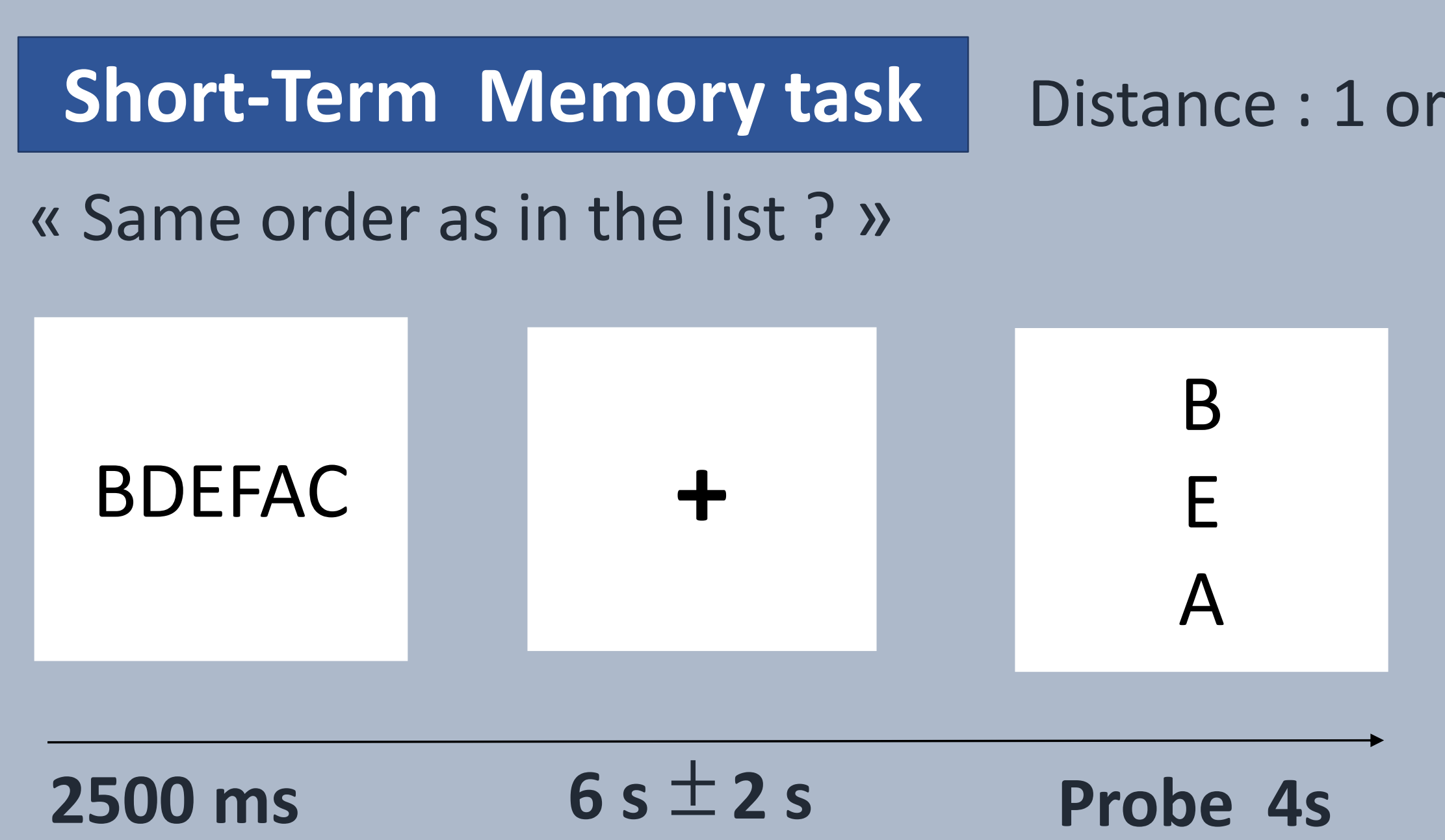

Luminance task Luminance distance : Close and far « Different? » Luminance distance :
Probe $4 \mathrm{~s}$ $+\quad \stackrel{7000 \pm 750 \mathrm{~ms}}{\longrightarrow}$
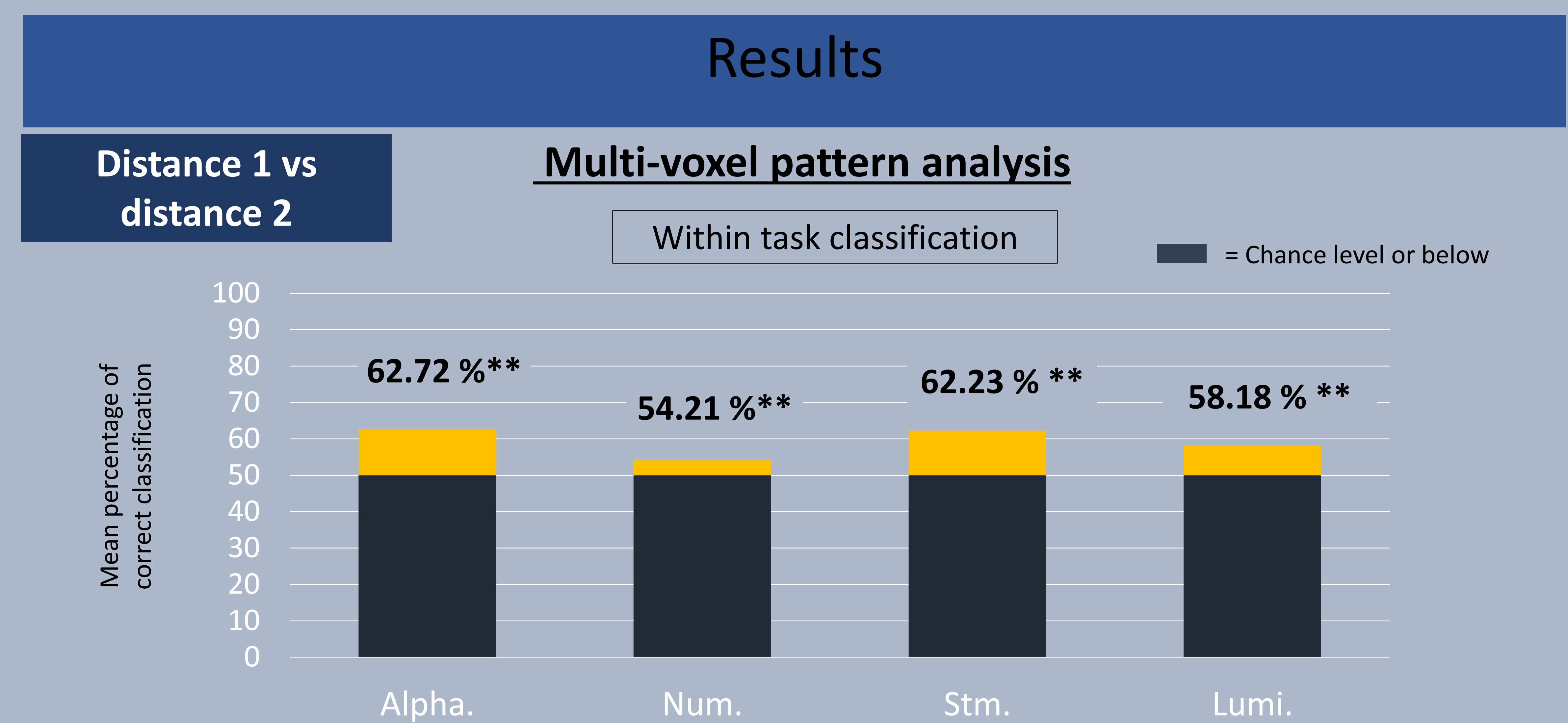

Whole brain within-task classification accuracy of ordinal distance $(* *=p<0.01)$

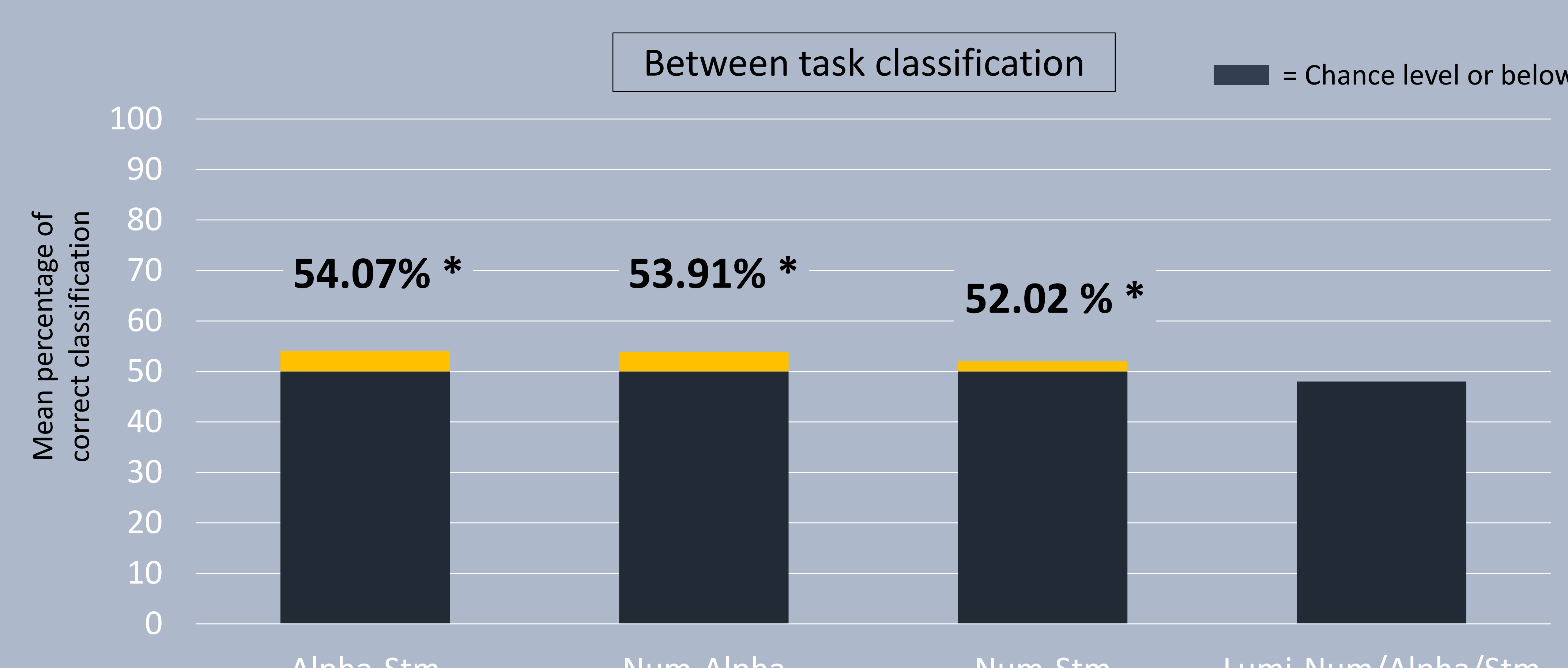

Whole brain between-task classification accuracy of ordinal distance $(*=p<0.05)$

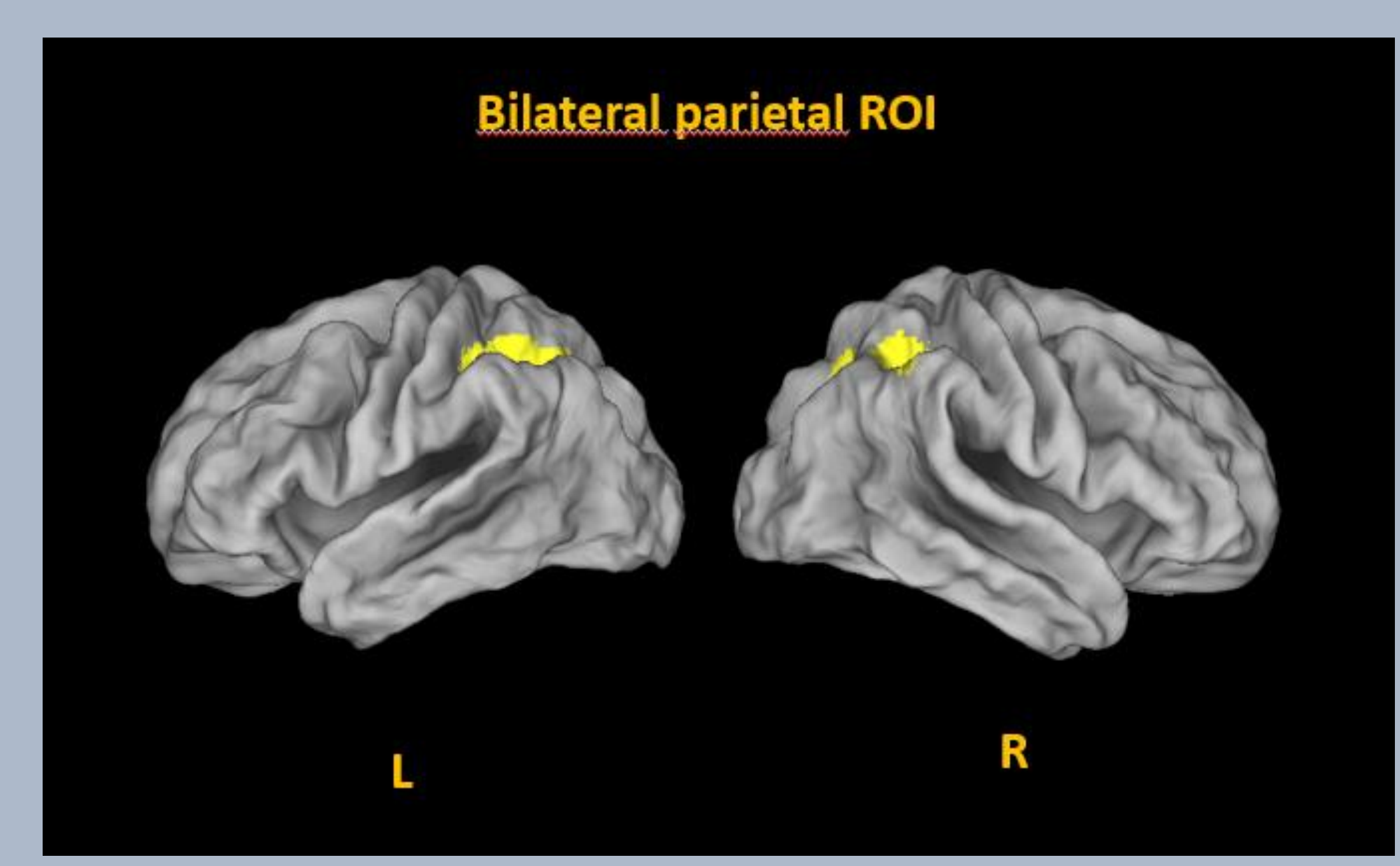

$\square$ The same results were observed when restricting the analyses to ROI in the bilateral intraparietal sulcus
- Robust ordinal distance effects observed for all tasks and greater-thanchance-level classifications when predicting ordinal distance between tasks.

- Involvement of a common serial order coding processes in the number, letter and working memory domains.

- Ordinal distance effects supported by intraparietal sulcus.

- Luminance distance could not be decoded by neural patterns for ordinal distance, indicating that the results are specific to ordinal processing, and not to distance per se.

\section{Conclusion}

Taken together, these results provide support for theoretical accounts considering the existence of domain general serial order coding processes.

Reference : Majerus, S., \& Attout, L. (2018). Working Memory for Serial Order and Numerical Cognition. In Heterogeneity of Function in Numerical Cognition (p. 409-431). https://doi.org/10.1016/B978-0-12-811529-9.00019-4 